\title{
Group Theory - a Tool to Analyse Infrared and Raman Spectra
}

\author{
S. Sudha* \\ Department of Physics, Bharath Institute of Higher Education and Research, Chennai-73, Tamil Nadu, India; \\ sudhavaidhy@gmail.com
}

\begin{abstract}
Group theory is a technique which helps spectroscopists to establish theoretical calculation in vibrational frequencies of a molecule. Vibrational frequencies of a molecule can be determined by Infra red and Raman spectroscopy. In this article, different symmetry elements in group theory has been discussed. Point groups have also been discussed. Using the symmetry elements determination of dipole moments of molecule, and optical activity of molecule have been done.
\end{abstract}

Keywords: Dipole Moment, Optical Activity, Point Group, Symmetry Element

\section{Introduction}

Group theory is a tool to treat invariants and symmetries of a molecule. Set of symmetry operations form a group. Symmetry operation is the movement of the molecule such that the resulting configuration of the molecule is indistinguishable from the original.

Symmetry element is a geometrical entity, such as a line or a plane or a point through which the symmetry operation like rotation or reflection or inversion is done. There is no translational motion of the molecule during the course of symmetry operation. Symmetry operations should be done within the molecule. All the symmetry elements intersect at a point which is unaffected by all the symmetry operations. For the particular molecule, when rotations or reflections take place, angular momentum of the molecule will remain invariant.

A molecule may have one or more of the symmetry elements, such as proper axis of symmetry $\left(\mathrm{c}_{\mathrm{n}}\right)$, plane of symmetry $(\sigma)$, improper axis of symmetry $\left(S_{n}\right)$, centre of symmetry (i) and identity element (E).

\section{Proper Axis of Symmetry $\left(C_{n}\right)$}

If $\theta$ is the smallest angle through which the rotation of a molecule is done with respect to an axis and if an indistinguishable configuration is got from that motion, the rotation is referred as a symmetry operation. The axis is known as proper axis of symmetry $\mathrm{C}_{\mathrm{n}} \cdot \mathrm{C}_{\mathrm{n}}$ denotes circular rotations around the axis, $\mathrm{n}$ represents the rotations performed successively to get a full circle. $n=360 / \theta$ and is called order of the symmetry axis.

If there are $C_{n}$ axes of different orders in a molecule, the axis with the highest order is referred to as principal axis Ex: Boron trichloride.

\section{Plane of Symmetry $(\sigma)$}

Plane, which bisects a molecule into two halves such that one is exactly the mirror image of the other, is said to be the reflection plane. This is also said to be plane of symmetry. The reflection planes can be classified into three types, based on their rotation with the principal axis. A plane is referred to as horizontal plane, $\left(\sigma_{\mathrm{h}}\right)$ if it is perpendicular

${ }^{*}$ Author for correspondence 
to the principal axis. A reflection plane, which contains the principal axis, is called vertical plane $\left(\sigma_{\mathrm{v}}\right)$. A vertical plane, which bisects two perpendicular $\mathrm{C}_{2}$ axes, is called dihedral plane $\sigma_{\mathrm{d}} \mathrm{Ex}: \mathrm{PtCl}_{4}$.

\section{Improper Axis of Symmetry $\left(S_{n}\right)$}

It is the line about which a rotation by a specific angle, followed by reflection in a plane perpendicular to the rotation axis is performed Ex: Ethane.

\section{Centre of Symmetry (i)}

This is a point in a molecule such that any line drawn through it meets the same atom at equal distances in opposite directions Ex: $\mathrm{H}_{2}$.

\section{Identity Operation (E)}

This operation leaves the molecule unchanged. It is denoted by the letter $\mathrm{E}$.

\section{Point Groups}

Set of symmetry elements of a molecule form point groups:

\section{1 $\mathrm{C}_{\mathrm{n}}$ Point Group}

A molecule, which possesses the proper axis of symmetry $C_{n}$ and the identity element, belongs to the $C_{n}$ point group.

\section{$7.2 \mathrm{~S}_{\mathrm{n}}$ Point Group}

A molecule, which has improper axis of symmetry $\left(S_{n}\right)$ and identity element, belongs to the $\mathrm{S}_{\mathrm{n}}$ point group.

\subsection{Dihedral $\left(\mathrm{D}_{\mathrm{n}}\right)$ Group}

A molecule, which has $\mathrm{C}_{\mathrm{n}}$ Principal axis and $\mathrm{n}$ perpendicular $\mathrm{C}_{2}$ axes, is denoted by $\mathrm{D}_{\mathrm{n}}$ point group.

\section{4 $\mathrm{D}_{\text {nd }}$ Point Group}

$\mathrm{D}_{\text {nd }}$ point group contains $\mathrm{C}_{\mathrm{n}}$ axis, $\mathrm{n}$ perpendicular $\mathrm{C}_{2}$ axes and $\mathrm{n}$ dihedral planes.

\section{5 $\mathrm{D}_{\mathrm{nh}}$ Point Group}

A molecule, which has $\mathrm{C}_{n}$ axis, $n$ perpendicular $\mathrm{C}_{2}$ axes and the horizontal plane of symmetry, comes under the $\mathrm{D}_{\mathrm{nh}}$ point group.

\subsection{Point Group}

A molecule, which possess the reflection plane and the identity element, belongs to the $\mathrm{C}_{\mathrm{s}}$ point group.

After determining point groups, the irreducible representations are formed from the characters of the reducible representations. The characters of the irreducible representation are tabulated in character table, which is used in determining the solution of molecular vibrations.

\section{Applications of Group Theory}

Group theory enables the determination of the fundamental frequencies, their degeneracies, the selection rule for the infra red and Raman spectra, the degrees of factors of the secular equation and the number of independent constants in the quadratic part of the potential function. It is found useful in analysing the splitting of over tone levels, possibility of perturbations due to resonance and to classify the normal modes of vibration according to the symmetry properties. It is important for the development of crystallography and condensed matter physics ${ }^{1-3}$.

\section{Dipole Moments}

The components $\mathrm{p}_{\mathrm{x}}, \mathrm{p}_{\mathrm{y}}, \mathrm{p}_{\mathrm{z}}$ of the electric dipole moment of a molecule along the Cartesian axes are given by

$$
\begin{aligned}
& \mathrm{p}_{\mathrm{x}}=\sum \mathrm{e}_{\mathrm{i}} \mathrm{x}_{\mathrm{i}} \\
& \mathrm{p}_{\mathrm{y}}=\sum \mathrm{e}_{\mathrm{i}} \mathrm{y}_{\mathrm{i}} \\
& \mathrm{p}_{\mathrm{z}}=\sum \mathrm{e}_{\mathrm{i}} \mathrm{z}_{\mathrm{i}}
\end{aligned}
$$

where, ei is the charge and $x_{i}, y_{i}, z_{i}$ are the coordinate of the ith particle. If $\mathrm{p}_{\mathrm{x}}=\mathrm{p}_{\mathrm{y}}=\mathrm{p}_{\mathrm{z}}=0$, the molecule has no permanent dipole moment, directed along one axis. If only one of the components is not zero, it has a permanent dipole moment directed along one axis which may be symmetry axis. If two components are non zero, permanent dipole moment is in a plane and if all three components are non zero, the dipole moment is not related to any one of the principal axis.

The dipole moment is a vector and must be totally symmetric. The components $\mathrm{p}_{\mathrm{x}^{\prime}}, \mathrm{p}_{\mathrm{y}^{\prime}}, \mathrm{p}_{\mathrm{z}}$ transform as $\mathrm{T}_{\mathrm{x}^{\prime}} \mathrm{T}_{\mathrm{y}^{\prime}}$ 
$\mathrm{T}_{z}$, respectively and, therefore, if any of the $\mathrm{T}_{\mathrm{x}}, \mathrm{T}_{\mathrm{y}}, \mathrm{T}_{\mathrm{z}}$ are totally symmetric, the corresponding components of the dipole moment can be non zero. These conditions requires that molecules having a permanent dipole moment may belong only to the point groups $\mathrm{C}_{1}, \mathrm{C}_{5}, \mathrm{C}_{\mathrm{n}}$ or $\mathrm{C}_{\mathrm{nv}}$.

In the $\mathrm{C}_{n}$ and $\mathrm{C}_{\mathrm{nv}}$ point groups, the dipole moment is along the $\mathrm{C}_{n}$ axis. In the $\mathrm{C}_{\mathrm{s}}$ point group, it lies in the plane of symmetry Ex: The water molecule belongs to the $\mathrm{C}_{2 \mathrm{v}}$ point group whose symmetry elements intersect in a line, the $\mathrm{C}_{2}$ axis. Therefore, the molecule has a permanent dipole moment along the $\mathrm{C}_{2}$ axis ${ }^{4,5}$.

\section{Optical Activity}

A molecule is said to be optically active, if it rotates the plane of polarized light passing through it. Consideration of symmetry properties for a molecule is useful in finding whether the molecule is optically active or not. If a molecule has $S_{n}$ axis of symmetry where $n=1,2,3$ it is not optically active ex:1, 1, Difluoro allene has a plane of symmetry and, so it is not optically active.

\section{Effects of Isotopic Substitution}

Electronic wave function for all isotopic species is taken as equivalent. The form of the electronic wave function is dependent on nuclear charge and the number of electrons in a molecule and not on nuclesr masses. Benzene and mono deutro benzene are isotopic species and they belong to $\mathrm{D}_{6 \mathrm{~h}}$ and $\mathrm{C}_{2 \mathrm{v}}$ point group respectively.

The electronic wave function of mono deutro benzene should be classified according to the $\mathrm{D}_{6 \mathrm{~h}}$ point group and not $\mathrm{C}_{2 \mathrm{v}}$. But vibrational wave functions are highly sensitive to nuclear masses and it should be classified according to $\mathrm{C}_{2 \mathrm{v}}$ point group.

\section{Conclusion}

Group theory has important applications in the study of molecular applications. It is much helpful in analysing the infrared and Raman spectra of polyatomic molecules, particularly in theoretical calculation. Group theory, together with fundamental ideas of spectroscopy, gives a fair idea about the understanding of the position and number of vibrational spectroscopic peaks. By having clear idea on the symmetry operations, one can understand group theory.

\section{Acknowledgement}

I am thankful to honourable chairman Pro-chancellor, Vice Chancellor, Registrar, Dean R\&D and Dean Engineering of Bharath University for their continuous support and encouragement given to carry out this research work.

\section{References}

1. Wilson EB Jr, Decius DC, Cross PC. Molecular vibrations. New York: McGraw Hill; 1955.

2. Woodward LA. Introduction to the theory of molecular vibrations and vibrational spectroscopy. Oxford University Press; 1972.

3. Raman KV. Group theory and its applications in chemistry Tenth reprint 2003. Tata McGraw hill co. New Delhi; 2003.

4. Hollos JM. Symmetry in molecules. London: Chapman and Hall Ltd.; 1972.

5. Dass NN. Symmetry and group theory for chemists. New Delhi: Asian Books Pvt Ltd.; 2004. 